\title{
The Poly Plot Package - Data Analysis Tools for Spectral Images
}

\author{
D. S. Bright and J. A. Small
}

Surface and Microanalysis Science Division, National Institute of Standards and Technology, Gaithersburg, MD. 20899-8371

Spectral images, now available from commercial instruments, present a large amount of data to the analyst. We have incorporated the Poly Plot Package (PPP) Tool (Figure 1) into Lispix to sort and sift the many spectra in this data and to extract the information we need [1].

Lispix reads and writes spectral images or cubes as raw data files (a contiguous block of binary data) and as Emispec* Series files. Spectrum images of about 128 x 128 x 1024 x 1 bytes comfortably reside in memory on a current PC. A small but growing set of the tools also work on much larger spectrum images (e.g. 512 × 512 × 2048 × 4 bytes) directly on the disk and use much less memory. These tools include reducing the cube by cropping the images, truncating the spectra and reducing the number of bytes per pixel.

A single spectrum (Figure 2) or a summed spectrum from the cube enables energy calibration using two peaks. Using this spectrum, you can select a peak, such as Al-Ka, to make an 'Aluminum' image (Figure 3). You can then choose any number of elements from the Periodic Table Tool, then the PPP Tool will make a stack of images for these elements summed from the appropriate energy ranges. (The elemental x-ray lines are part of the Periodic Table Tool). You can then threshold and blob any of these images to locate particles (Figure 3) that are high in that element. The Blob Tool (Figure 4) can make a table or spreadsheet with one row for each blob and with columns for size, location, morphology and x-ray counts of selected elements. The Spreadsheet Tool is useful for plotting data, and selecting spectra or particles. Selections transfer to and from the Blob tool for locating particles in an image, and to and from the PPP tool for examining their spectra.

Many spectra are hard to compare using plots, but are easier to compare using the PPP image (Figures 5,6). This image displays one spectrum per line using an adjustable thermal scale. Heights of lines can be zoomed up to x8 (shown here). Like spectrum plots, the PPP image may have x-ray line markers. In addition, the spectra can be sorted and selected. It is practical to show several hundred spectra at once in the PPP image, which can be scrolled up and down to view them. All of the spectra in the cube can be sorted individually, and the top ones displayed in the PPP image.

Other useful functions include: scatter diagrams and RGB color overlays of two or three elemental images, Principal Component Analysis of images (not spectra), smoothing of images, and image enhancement. Blobs selected from a data cube can be related to objects in another image of the same field, such as a backscatter image taken at higher resolution. The PPP tool can correct for offsets, rotation and changes in scale between the cube and the high resolution image.

Future development includes making k-ratio cubes, handling of sparse arrays (spectrum cubes with most pixels blank, spectra in the rest), and cube i/o in XML format some other standardized format..

\section{References:}

[1] The PPP is a tool included in Lispix, a public domain image processing system for the PC. Lispix is available for download at www.nist.gov/lispix.

* Certain commercial equipment, instruments, or materials are identified in this report to specify adequately the experimental procedure. Such identification does not imply recommendation or endorsement by the National Institute of 
Standards and Technology, nor does it imply that the materials or equipment identified are necessarily the best available for the purpose.

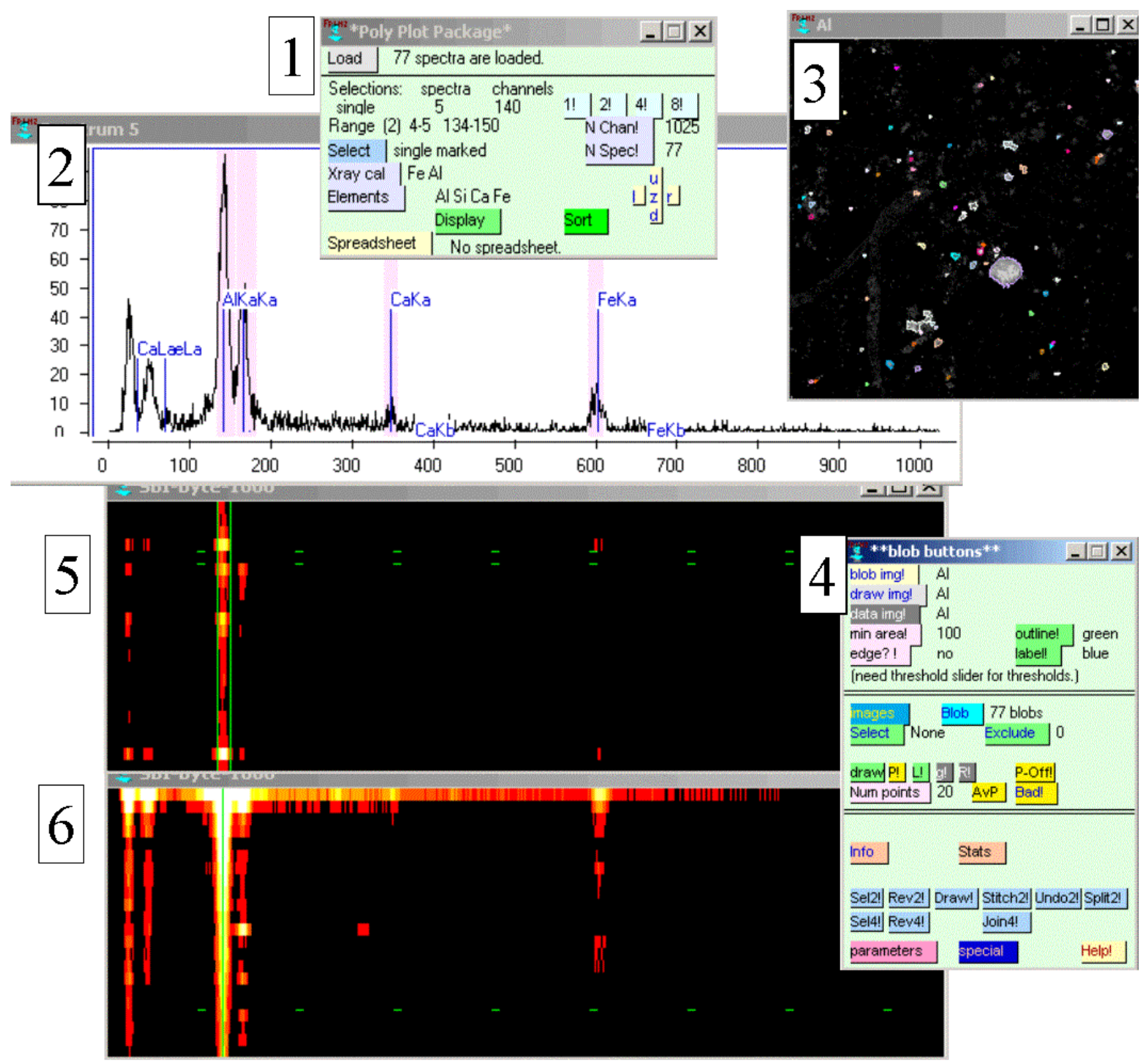

FIG. 1. PPP Tool. 77 spectra correspond to 77 blobs in Figure 4.

FIG. 2. Plot of spectrum. $\mathrm{x}$ axis - channel number. Y axis - counts.

FIG. 3. 'Aluminum' image with counts under the. Al-K $\alpha$ peak.

FIG. 4. Blob Tool. 77 spectra correspond to 77 particles in PPP tool (Figure 4).

FIG. 5. PPP image. Channel range selected for sort shown in Figure 6.

FIG. 6. PPP image after sorting on range in Figure 5. Dashed green line - spectrum in Figure 5. 